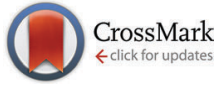

Cite this: J. Mater. Chem. C, 2016, 4, 1550

Received 12th October 2015, Accepted 23rd January 2016

DOI: $10.1039 / c 5 t c 03281 d$

www.rsc.org/MaterialsC

\title{
Organic energy devices from ionic liquids and conducting polymers $\dagger$
}

\author{
Robert Brooke, ${ }^{\text {ab }}$ Manrico Fabretto, ${ }^{\mathrm{b}}$ Marta Krasowska, ${ }^{\mathrm{b}}$ Pejman Talemi, ${ }^{\mathrm{bc}}$ \\ Samuel Pering, ${ }^{d}$ Peter J. Murphy ${ }^{\mathrm{b}}$ and Drew Evans*b
}

\begin{abstract}
The use of smart technologies in our daily lives, from smartphones to auto-dimming windows to touch sensors, has become pervasive. With growing desire for these devices to be conformable and flexible, traditional materials are being replaced to create a class of products known as active organic electronic devices (OEDs). These new devices owe their ability to switch electrical and/or optical function to the intimate interaction between an inherently conducting polymer and electrolyte, typically an ionic liquid. Herein, we provide the first observations that specific ionic liquids can reduce or oxidise conducting polymers upon intimate contact in the absence of any electrical stimuli. The ability to reduce or oxidise the inherently conducting polymer depends on the cation and anion pair within the ionic liquid. Extending the utility of this phenomenon is made by fabricating OEDs such as prototype fuel cells, supercapacitors and smart windows.
\end{abstract}

\section{Introduction}

From generating light, ${ }^{1}$ to observing the universe ${ }^{2}$ to storing electronic data ${ }^{3}$ and electrical energy, ${ }^{4}$ inherently conducting polymers (ICPs) and ionic liquids (ILs) are independently in their own rights materials with seemingly ubiquitous use in a diversity of applications. The rapidly growing field of flexible organic electronic devices (OEDs), ${ }^{5,6}$ and in particular active OEDs, embody technologies that utilise the intimate interaction between these two materials. However, despite the discovery of ICPs in the $1970 \mathrm{~s}^{7}$, and to an even greater extent the development of ILs over the past 125 years, ${ }^{8,9}$ their interaction has scarcely been discussed. ${ }^{10,11}$ In most of the electro-chemically active OEDs the IL is simply employed as an electrolyte providing both cations and anions to facilitate the (reversible) electrochemical reduction or oxidation of the ICP. The novelty of using an IL here extends from the fact that the cations and anions always exist in solution, in contrast to classic electrolytes which only generate ions through dissociation or ionisation in the

\footnotetext{
${ }^{a}$ Linkoping University, Department of Science and Technology, Organic Electronics, SE-601 74 Norrkoping, Sweden

${ }^{b}$ Future Industries Institute, University of South Australia, Mawson Lakes, South Australia, 5095 Australia. E-mail: drew.evans@unisa.edu.au

${ }^{c}$ School of Chemical Engineering, University of Adelaide, Adelaide, South Australia, 5001 Australia

${ }^{d}$ Department of Chemistry, University of Bath, Bath, BA2 7AY, UK

$\dagger$ Electronic supplementary information (ESI) available: (1) Detailed experimental methods, (2) atomic Force Microscope experimental data, (3) X-ray Photoelectron Spectroscopy analysis, (4) optical memory experiments, (5) smart window and supercapacitor prototype devices. See DOI: 10.1039/c5tc03281d
}

presence of a solvent. ${ }^{12}$ Such oxidation or reduction leads to modification of the ICPs electrical and optical properties, thus creating active OEDs such as electrochromic smart windows and displays, ${ }^{13}$ organic electrochemical transistors (OECT) and sensors, ${ }^{14}$ and electrochemical mechanical actuators. ${ }^{10}$ Prior research on the ICP-IL interaction has been heavily focussed on how the cations and/or anions diffused in and out of the ICP, with $^{15,16}$ or without ${ }^{11}$ an externally applied electric field.

Within this context, the fields of chemistry and biology commonly apply theories such as the Hard-Soft Acid-Base (HSAB) theory ${ }^{17}$ and Marcus theory ${ }^{18}$ to understand and categorise how and why specific chemicals and molecules interact and/or react. The kinetics of reactions involving electron transfer between molecules and/or atoms, including the solvent medium is well described by Marcus Theory. Note that there is also the scale of Gutmann which defined a donor and acceptor number, ${ }^{19}$ which ultimately translated the acceptor and donor character of solvents and molecules into a relative scale. These numbers then define for molecules, such as ILs and ICPs, the direction of the electron transfer (leading to reduction or oxidation). To date, general observation of this charge transfer has not been reported.

Examination of the literature reveals one comment on the potential use of the HSAB theory to describe the interaction of anions with ICPs. ${ }^{20}$ Garnier et al. state that the HSAB theory presents an interesting mechanism to interpret how ICPs interact with their doping counter ion (the anions responsible for stabilisation of the carbo-cation in the ICP). Crispin et $a .^{21}$ in complementary work also discussed ICPs $(\pi$-conjugated molecules) in this context, focussing on the interaction of acetonitrile with transition metal layers such as $\mathrm{Cu}, \mathrm{Ni}$ and $\mathrm{Fe}$. With respect 
to the use of OECTs ${ }^{22,23}$ for chemical and/or biological sensing (e.g. detection of glucose ${ }^{14}$ ), the specific interaction between the ICP and the electrolyte will need to be taken into account prior to unravelling the manner by which the analyte interacts with the ICP. As this literature survey suggests, to date no general practical demonstration has been made for ILs reducing or oxidising ICPs. Of note though is the work of Armel et al. who observed one specific IL changing the colour (darkening) of poly(3,4-ethylenedioxythiophene) (PEDOT), though they concluded no electrical reduction of the ICP occurred. ${ }^{11}$ General observations in the presence of an external electric field have been made by Forsyth and co-workers showing that both the nature of the anion and cation influence the redox cycling of electrodeposited conducting polymer films. ${ }^{24}$ This is supporting evidence that the chemistry of an IL should be considered in defining the performance of an OECD.

Herein we utilise the fact that ILs and ICPs can be selected to interact and lead to reduction or oxidation of the ICP (based upon the aforementioned mechanisms described by HSAB, Marcus theory, Gutmann number, etc.). Firstly, we present experimental observations that upon intimate contact when ILs diffuse into ICPs they can reduce, oxidise or do nothing to the ICP, all in the absence of an externally applied electric field. In this study the different redox behaviour observed for given ILs and PEDOT are summarised and categorised. We use these redox interactions to fabricate several different types of OEDs consisting of only ILs and ICPs such as PEDOT; (i) electrochromic displays; (ii) smart windows, (iii) supercapacitors, (iv) fuel cells.

\section{Results and discussion}

Of the many ICPs currently being investigated, ${ }^{5}$ PEDOT and more specifically PEDOT doped with tosylate (Tos) anions, synthesised using vapour phase polymerisation (VPP), is considered to be the one with the greatest potential for ubiquitous use. It has recently been demonstrated to be semi-metallic ${ }^{25}$ with high electrocatalytic activity, ${ }^{26,27}$ and possessing good electrochromic switching ${ }^{13}$ and biocompatibility. ${ }^{28}$ Herein, PEDOT-Tos (and PPy-Tos and PTh-Tos) was prepared by VPP and employed as thin films on glass substrates to study its interaction with a variety of ILs. The PEDOT has a high doping level with Tos approaching one Tos for every 3 EDOT repeat units to achieve a high electrical conductivity $\left(>1500 \mathrm{~S} \mathrm{~cm}^{-1}\right)$. This conductivity is primarily attributed to the many positive charges positioned along the conjugated polymer backbone. ${ }^{13,25}$ The interaction of these doped conducting polymers with a range of ILs, without the application of an external electric field, is shown in Table 1.

Interaction of a variety of ILs with PEDOT-Tos is observed as an optical darkening, or reduction (Table 1), in a similar fashion to the change in properties observed when interacting with other molecules known for their hard base character (for example amines ${ }^{29-31}$ ). When trihexyl(tetradecyl) phosphonium bis(2,4,4-trimethylpentyl)phosphinate (THTDPh-BisPhos) contacts the PEDOT-Tos it reduces, while 1-butyl-3-methyllimidazolium bis(trifluoromethylsulfonyl)imide (BMIM-TFSI) and other similar
Table 1 The visual observation of either no response, or chemical reduction, when specific ILs are placed into intimate contact with ICPS in the absence of an applied electric field. THTDPh-BisPhos is observed to reduce PEDOT, PPy and PTh

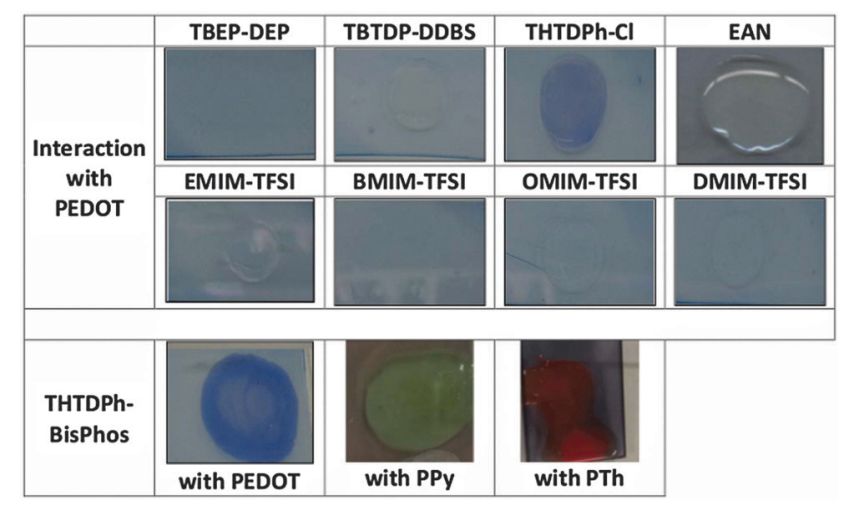

ILs do not reduce the ICP (Table S1, ESI $\dagger$ ). The observation of chemical reduction is also made for THTDPh-BisPhos in contact with PPy-Tos and PTh-Tos. In each case the IL is hypothesised to displace the doping Tos anion from within the PEDOT. This is substantiated by the introduction of $\mathrm{Cl}$ as the dopant anion (VPP PEDOT using $\mathrm{FeCl}_{3}$ as the oxidant), which significantly inhibited the reduction reaction when contacted with the same IL. The rationale here is that $\mathrm{Cl}$ is strongly bound within the PEDOT due to its strong base character, while Tos is not so strongly bound. This highlights that the chemical character of the materials is just as important as the electrostatic interactions (i.e. anions attracted to cations). The importance of the specific chemistry in the system highlights the usefulness of theories such as the HSAB and Marcus theory for IL-ICP interactions. Much of the literature focusses on the physics of these interactions, discussing in detail electrostatic interaction of like and dissimilar charged species.

To confirm the specific interactions between the ICP and the ILs, including those that did not appear to show observable colour change, the thickness of the PEDOT-Tos was measured before and after contact with the IL for several ICP-IL examples. The BMIM-TFSI gave a $34 \%$ thickness increase and the 1-butyl-

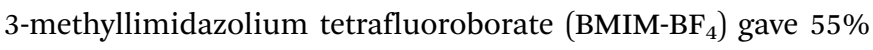
(see ESI $\dagger$ ). The swelling magnitude compares favourably to the percentage increase reported by Armel $e t a l .{ }^{11}$ for concentrated phosphonium cation-Tos anion ILs in direct contact with similar VPP PEDOT-Tos substrates. Their investigation concluded that the swelling arose from the uptake of the IL into the PEDOT-Tos, leading to an expansion of the $d 100$ interchain distance. Expansion due to the large phosphonium cation altered the PEDOT interchain interactions, with the observation of a concomitant optical darkening of the PEDOT-Tos. Their electrical analysis suggested this darkening was not due to a chemical reduction of the PEDOT-Tos. Our observation of comparable polymer swelling for VPP PEDOT-Tos upon contact with the TFSI and $\mathrm{BF}_{4}$ based ILs without any change in visual appearance/colour suggests that there are additional factors at play beyond what was proposed by Armel et al. ${ }^{11}$ 
(a)

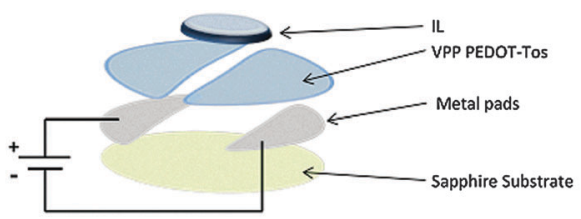

(b)

To investigate if the specificity of the IL is important under an applied electric field, high doped PEDOT-Tos samples were scored in half to create two electrodes (Fig. 1a), essentially creating an anode and cathode. Electrochemical reduction or oxidation was initiated by applying an electrical field between the two polymer electrodes in the presence of selected ILs. Hence one electrode underwent oxidation while the other reduced, and with reversal of the bias the PEDOT-Tos changed to its opposite redox state (Fig. 1b). For certain ILs there was observable optical switching of the polymer, in agreement with previous studies, ${ }^{32,33}$ even after multiple switches back and forth between the two states. In situ to the AFM, the PEDOT-Tos electrodes are still observed to optically switch between the reduced and oxidised states (Fig. 1c), with concomitant changes in the measured film thickness (Fig. 1d). After switching between the reduced and oxidised state several (up to 20) times using the TFSI anion, a further swelling of the PEDOT-Tos ( $\mathrm{ca}$. an additional 20\%) was recorded relative to the initial swelling upon contact without applying a bias.

However in contrast, the use of the THTDPh-BisPhos IL yielded no change in the PEDOT-Tos film thickness. In fact, even with the application of an external bias that should oxidise the PEDOT-Tos, it remained in the reduced state as observed by the unchanged dark optical appearance. Observations here add

to the growing discussion that an IL is not simply described as a "sea of point charges", ${ }^{34-36}$ nor a homogeneous continuum of non-interacting dipoles, ${ }^{37}$ rather their specific chemistry is important to how ILs behave and interact with other materials. As will be shown herein, this has direct implication for applications such as fuel cells, supercapacitors and smart windows.

\section{Electrochromic devices}

We have already visually observed in Table 1 that certain ILs reduces PEDOT-Tos (and PPy and PTh). Further to this, using UV-Vis spectroscopy, when ethylammonium nitrate (EAN) is used it oxidises the PEDOT-Tos (Fig. 2). This oxidation is confirmed by observing an increase in the optical transmission of the PEDOT-Tos (this transparency is difficult to identify in the optical images presented in Table 1). In Fig. 2, the electrochromic properties of PEDOT-Tos with EAN is compared with that of BMP-TFSI which has been previously employed for active OEDs. ${ }^{13,38,39}$ The PEDOT-Tos with no applied external electric field in contact with the BMP-TFSI is less transparent than the oxidised state (i.e. $+1.5 \mathrm{~V}$ bias applied to the PEDOT-Tos). Converse to the expected response, when using EAN as the electrolyte, both the no bias and oxidised states of the PEDOT-Tos are in perfect agreement (highly transparent). This implies that the EAN strongly oxidises the PEDOT-Tos without an externally applied electric field. Given the strong oxidation strength of the EAN, it is employed to facilitate the oxidative polymerisation of EDOT (Fig. 2d), by adding EDOT monomer directly to the IL in the absence of any other chemical. The increasing blue colour with time of the initially transparent liquids confirms the oxidative polymerisation of EDOT using only an appropriate IL. The oxidation properties of the EAN is hypothesised to arise from the IL only, and not dissolved gases within (such as oxygen), as supported by the observation of the same chemical oxidative polymerisation of EDOT in EAN under vacuum (an oxygen deficient environment). In a similar manner to the lack of film thickness change upon electrochemical reduction or oxidation for THTDPhBisPhos, EAN initially swelled the ICP upon contact but no further change upon cycling.

The chemical reactions between the IL and ICP are hypothesised to be driven by the chemistry of the IL cations $(\mathrm{N}+$ or $\mathrm{P}+)$ and the relative 'strength' of the anion (categorised as a base). Firstly, the anions in the IL can be defined as a base as per the HSAB theory. In the case of $\mathrm{NO}_{3}-$ and $\mathrm{Cl}-$, these hard base anions lead to redox reactions with the ICP; when paired with $\mathrm{N}+$ or $\mathrm{P}+$ cations they oxidise or reduce the ICP respectively. Conversely, soft base anions such as TFSI-, $\mathrm{BF}_{4}-$ and $\mathrm{PF}_{6}-$ are inert irrespective of which cation they are paired with. This very simplistic description of the ILs serves as a starting point upon which to apply the IL-ICP reactions in devices and a foundation upon which to understand the mechanism in detail.

From the comparison of the two ILs, the oxidised state achieved with the BMP-TFSI is not as transparent ( 0.25 absorbance at $550 \mathrm{~nm})$ as the no bias or oxidised states $(0.2$ absorbance at $525 \mathrm{~nm}$ ) observed for that with EAN. This highlights that the oxidation level achieved through external electrical stimulus using BMP-TFSI is less than that achieved by chemical oxidation 
(a)

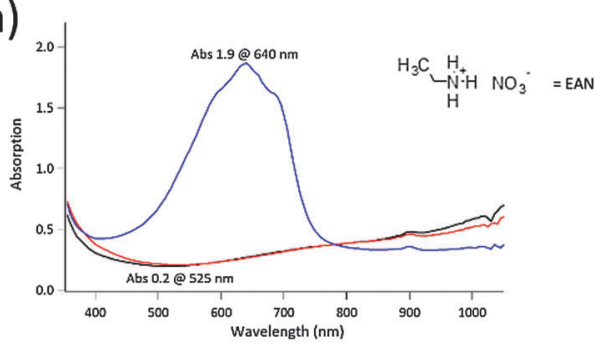

(b)

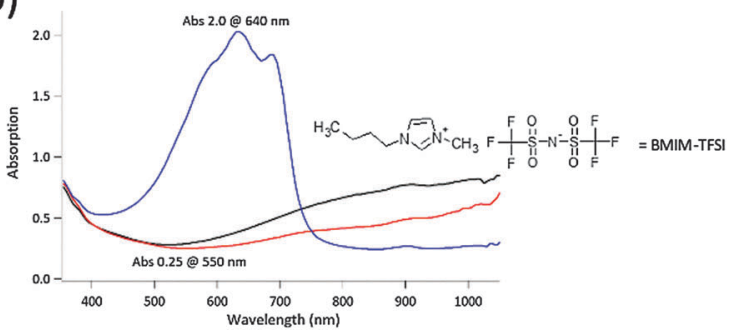

(c)
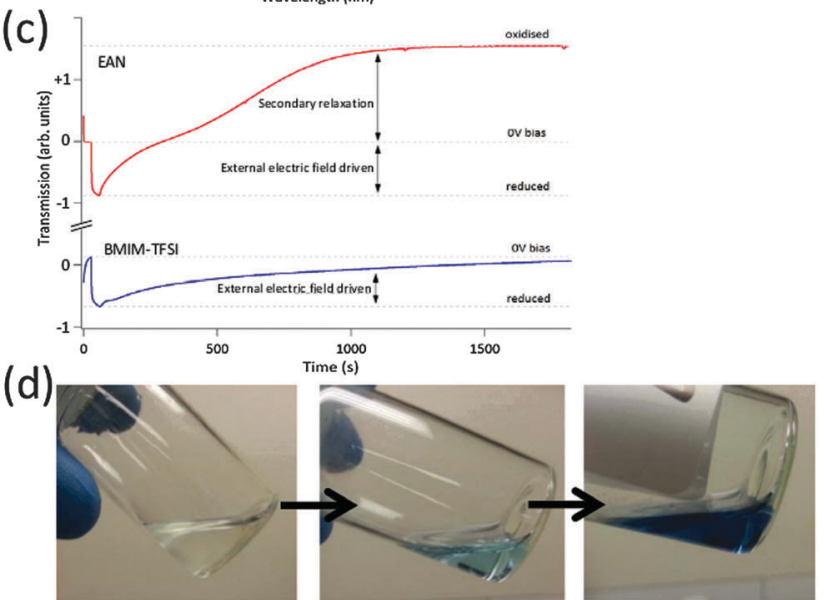

Fig. 2 The optical switching of ICPs in various ILs as measured by absorption UV-Vis spectroscopy. (a) The neutral (black), reduced (blue) and oxidised (red) states of PEDOT:Tos when EAN is employed. (b) The neutral (black), reduced (blue) and oxidised (red) states of PEDOT:Tos when BMIM-TFSI is employed. The maximum and minimum absorption values for the reduced and oxidised state are reported for each IL in (a) and (b). (c) The relaxation of PEDOT-Tos from its switched state, back to its neutral state when the system is in open circuit (known as optical memory). (d) The oxidative polymerisation of EDOT monomer to form PEDOT using only a hard acidhard base IL.

using EAN. Such an observation further highlights that the chemical nature of the IL and ICP is important in defining their interaction.

\section{Optical memory}

The phenomenon displayed in Fig. 3c, slow relaxation of optical properties after disconnecting the electrical stimuli, is commonly referred to as "optical memory" ${ }^{40-42}$ This refers to the ICPs ability to retain its switched optical state for extended periods of time once the externally applied electric field is removed (the external power source is disconnected, i.e. open circuit). The decay in optical properties to the neutral state from either the reduced or oxidised state shown in Fig. $2 \mathrm{c}$ were made in a test cell (Fig. S13, ESI $\dagger$ ), having an electrode separation of several mm,
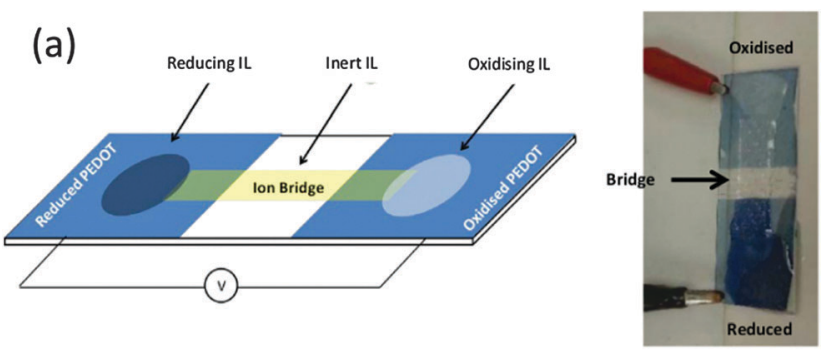

(b)
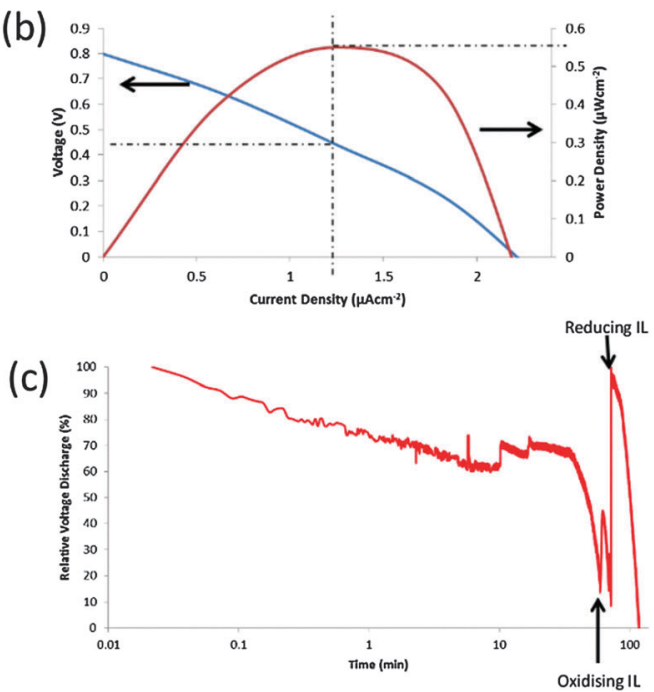

Fig. 3 (a) The architecture of the prototype metal-free fuel cell. One PEDOTTos electrode is reduced, the other is oxidised, and they are bridged with an ion channel using the inert IL. An example of the working ICP-IL fuel cell is presented, showing the reduction and oxidation occurring at each half of the cell. (b) The power output for the prototype device is $0.5 \mu \mathrm{W} \mathrm{cm}{ }^{-2}$ or $31 \mathrm{~mW} \mathrm{~g}^{-1}$ of PEDOT-Tos, with (c) the output voltage being recovered after discharge by introduction of more IL "fuel".

without full encapsulation of the polymer electrodes and IL. For the TFSI and $\mathrm{BF}_{4}$ based ILs the optical memory can be as long as several hours. In contrast, the EAN system has an optical memory relaxing from the reduced to the no bias state of the order of only several minutes. These different electrochromic behaviours for the different ILs using the same ICP represent advances for practical applications of such devices. Firstly, the long optical memory obtained for the TFSI and $\mathrm{BF}_{4}$ based ILs is suitable for low power consumption "smart window" applications such as architectural glazing, where a short voltage pulse every few hours is sufficient to keep the device in the desired optical state. In contrast, the short optical memory returning to the oxidised state for the EAN system is suitable for applications requiring a safety fail-safe such as dimmable automotive rear view mirrors (a power failure returns it to a conventional reflective mirror).

Based on this knowledge, we fabricated a series of prototype devices that demonstrate the impact of the ICP-IL interaction for energy related applications.

\section{Smart window}

A smart window (Fig. S15, ESI $\dagger$ ) was fabricated in which BMPTFSI was encapsulated in a working active OED with high 
doped PEDOT-Tos as the working electrode, and PPy as the counter electrode (both electrodes were deposited onto ITO coated glass substrates). The resulting device has a persistence of the switched state out to greater than $12 \mathrm{~h}$. Conversely, devices prepared using THTDPh-BisPhos did not function, and those using EAN persisted in the switched state for only a few hours. It is noted that the change in electrochromic cell geometry from Fig. S13-S15 (ESI $\dagger$ ) leads to much longer optical memory of the device. Also, these are significantly longer times than those reported for other electrochromic devices. ${ }^{40,43}$

\section{Supercapacitors}

A symmetric supercapacitor device using solely conducting polymers (commonly known as Type $\mathrm{I}^{44}$ ) was fabricated using high doped PEDOT:Tos as both electrodes, and different ILs as the spacer medium. Through changes in the IL chemistry, the specific capacitance of the supercapacitor was increased by an order of magnitude (Fig. S16, ESI $\dagger$ ), despite these ILs being estimated to have comparable dielectric constants. ${ }^{37}$ Using BMP-TFSI yielded a capacitance of $c a .70 \mathrm{~F} \mathrm{~g}^{-1}$ by weight of the two PEDOT electrodes, compared to only $1 \mathrm{~F} \mathrm{~g}^{-1}$ for the THTDPh-BisPhos, at a voltage scan rate of $100 \mathrm{mV} \mathrm{s}^{-1}$. The highest value herein compares favourably to other symmetric PEDOT based supercapacitors such as those of Kaner and co-workers for structured PEDOT electrodes ${ }^{45}$ and Yang et al. for bulk PEDOT electrodes. ${ }^{46}$ However these results were achieved using high concentration $\mathrm{HCl}$ or $\mathrm{H}_{2} \mathrm{SO}_{4}$ electrolytes, as opposed to the simple ILs herein. Conversely the PEDOT-based supercapacitors fall short of the capacitance values obtained for protic ILs used with carbonaceous electrodes $\left(>120 \mathrm{~F} \mathrm{~g}^{-1}\right) .{ }^{47}$ Significant modification of the specific capacitance for this simple supercapacitor demonstrates how the knowledge of chemical interactions of ILs and ICPs can inform the design of future energy storage devices.

\section{Fuel cell}

Deviating from capacitor and electrochromic properties (storage of charge), we show the relevance of the ICP-IL interactions for the fabrication of a fuel cell that generates power solely based on the ICP-IL interactions. The prototype fuel cell device in Fig. 3a uses a THTDPh-BisPhos to reduce PEDOT-Tos, and EAN to oxidise the same PEDOT-Tos. These two half cells are bridged using a TFSI based IL to create an ion conductive bridge, in a configuration typical of a galvanic cell. Such prototype devices yield voltages of up to $1 \mathrm{~V}$ across the two PEDOT electrodes (electrodes fabricated from the same PEDOT thin film). The mechanism by which this fuel cell operates is not understood. However, it is hypothesized that the IL anion is intercalating within the PEDOT-Tos structure, in a manner similar to the intercalation of Li cations in a Li-ion battery. Analysis (Fig. 3b) of the fuel cell performance reveals the fuel cell generates up to $0.5 \mu \mathrm{W} \mathrm{cm}^{-2}$ (ca. $30 \mathrm{~mW} \mathrm{~g}^{-1}$ of PEDOT-Tos) of power, at $0.45 \mathrm{~V}$ and $1.25 \mu \mathrm{A} \mathrm{cm}^{-2}$. Measurement of a non-zero power density for this symmetric cell (i.e. both electrodes are the same material) opens the possibility for an ICP-IL redox flow battery to be fabricated. Such non-aqueous redox batteries are of interest owing to the potential for higher energy density devices. ${ }^{48}$ The definition of a fuel cell arises from the fact that during discharge experiments (Fig. 3c) the addition of more IL (fuel) leads to a sharp increase in voltage, highlighting the IL is being "consumed" in this process. Employing only ICPs and ILs to create a metal-free fuel cell highlights the potential impact the new knowledge of ICP-IL interactions will have on next generation energy-related devices.

\section{Conclusion}

In summary, we have demonstrated that ILs can oxidise or reduce ICPs in a similar manner to observations of chemical redox reactions with acids/bases and ICPs. Interaction between the two can be viewed as a combination of processes driven by the chemical nature of the IL and ICP, and any externally applied electric field. These interactions can be categorised as, hard base anions ( $\mathrm{NO}_{3}-$ and $\mathrm{Cl}-$ ) induce oxidation or reduction of the ICP when paired with $\mathrm{N}+$ or $\mathrm{P}+$ cations respectively. Employing soft base anions (TFSI, $\mathrm{BF}_{4}, \mathrm{PF}_{6}$ ) results in no observable chemical reaction irrespective of which cation is utilised. The applicability of this new knowledge extends the manner by which optical switching of ICPs is now understood, and furthermore it helps explain the relaxation processes involved with the commonly observed optical memory phenomenon for ICPs. Implementing this into prototype devices (fuel cell, supercapacitors and smart windows) has demonstrated excellent performance, with the case of the novel fuel cell achieving voltages up to $1 \mathrm{~V}$, and a power output of $c a .0 .5 \mu \mathrm{W} \mathrm{cm}{ }^{-2}$. We believe the observations of redox reactions between ILs and ICPs will help to unlock future opto-electronic device performance, leading to the creation of efficient novel organic electronic devices.

\section{Experimental section}

\section{Materials}

The ionic liquids, 1-butyl-1-methylpyrrolidinium bis(trifluoromethylsulfonyl)imide (BMP-TFSI), 1-ethyl-3-methylimidazolium bis(trifluoromethylsulfonyl)imide (EMIM-TFSI), 1-butyl-3-methyllimidazolium bis(trifluoromethylsulfonyl)imide (BMIM-TFSI), 1-octyl-3-methylimidazolium bis(trifluoromethylsulfonyl)imide (OMIM-TFSI), 1-decyl-3-methylimidazolium bis(trifluoromethylsulfonyl)imide (DMIM-TFSI), 1-dodecyl-3-methylimidazolium bis(trifluoromethylsulfonyl)imide (DDMIM-TFSI), tributylethylphosphonium diethyl phosphate (TBEP-DEP), tributyl(tetradecyl)phosphonium dodecylbenzenesulfonate (TBTDP-DDBS), ethylammonium nitrate (EAN), 1-butyl-3-methyllimidazolium tetrafluoroborate (BMIM-BF 4 ), trihexyl(tetradecyl) phosphonium bis(2,4,4-trimethylpentyl)phosphinate (THTDPh-BisPhos) and trihexyl(tetradecyl) phosphonium chloride (THTDPh-Cl) were purchased from Iolitec and were of at least 98\% purity. 3,4-Ethylenedioxythiophene (EDOT, Clevios V2) and iron tosylate $\left(\mathrm{Fe}(\mathrm{Tos})_{3}\right.$, Clevios CB-40 V2) were purchased from Heraeus. Poly(ethylene glycol)-poly(propylene glycol)-poly(ethylene glycol) (PEG-PPG-PEG, Pluronic P-123), $M_{\mathrm{w}}=5800 \mathrm{~g}$ mol and lithium 
perchlorate was obtained from Aldrich. The ionic liquids were purified before use. All other chemicals were used as received.

\section{Sample preparation}

PEDOT, polypyrrole (PPy) and polythiophene (PTh) were fabricated via VPP using $\mathrm{Fe}(\mathrm{Tos})_{3}$ as the oxidant in butanol solvent, with added PEG-PPG-PEG triblock copolymer (5800 Da $M_{\mathrm{w}}$ ) to create high doped PEDOT (see ESI $\dagger$ ). These ICP substrates were exposed to a variety of ILs; namely BMP-TFSI, BMIM-TFSI, EAN, BMIM-BF $_{4}$, THTDPh-BisPhos and THTDPh-Cl.

\section{Analysis}

Atomic force microscopy was employed to determine the change in thickness of the high doped PEDOT-Tos, comparing the 'in-air' thickness with that upon exposure to the IL, and upon electrochemical reduction or oxidation due to an applied bias (see Fig. S1-S5, ESI $\dagger$ ). Importantly the thickness is measured in-situ when fully immersed in the IL, avoiding capillary forces and effects coming from static electrostatic charge on the tip and the sample, minimising adhesion force between the AFM tip and the ICP substrate, and thus avoiding significant error in the thickness measurement. The electrochromic behaviour of the high doped PEDOT-Tos in combination with the different ILs was tested using a test cell consisting of the PEDOT-Tos as one electrode, ITO as the counter electrode, and the IL sandwiched between as the electrolyte (Fig. S13, ESI $\dagger$ ). Optical transmission was measured using a HunterLab UV-Vis spectrophotometer, while a LED/Photodiode detector pair was used to determine the change in transmission versus time for optical memory measurements. The prototype fuel cell was fabricated from high doped PEDOT:Tos, which was cut into two electrodes. Each electrode was exposed to either EAN or THTDPh-BisPhos, and the two halves of the cell bridged using a cellulose fibre mat presoaked with BMIM-TFSI (an inert ion bridge). This cell was interrogated using an EZstat-Pro potentiostat/ galvanostat (NuVant System Inc.) to measure the current density and power density of the fuel cell.

\section{Acknowledgements}

The authors acknowledge Kamil Zuber for assistance with optical measurements. This work was performed in part at the South Australian node of the Australian National Fabrication Facility, a company established under the National Collaborative Research Infrastructure Strategy to provide nano- and micro-fabrication facilities for Australia's researchers.

\section{Notes and references}

1 T.-H. Han, Y. Lee, M.-R. Choi, S.-H. Woo, S.-H. Bae, B. H. Hong, J.-H. Ahn and T.-W. Lee, Nat. Photonics, 2012, 6, 105.

2 E. F. Borra, O. Seddiki, R. Angel, D. Eisenstein, P. Hickson, K. R. Seddon and S. P. Worden, Nature, 2007, 447, 979.

3 S. Möller, C. Perlov, W. Jackson, C. Taussig and S. R. Forrest, Nature, 2003, 426, 166.
4 M. Armand, F. Endres, D. R. MacFarlane, H. Ohno and B. Scrosati, Nat. Mater., 2009, 8, 621.

5 S. R. Forrest, Nature, 2004, 428, 911.

6 M. F. El-Kady, V. Strong, S. Dubin and R. B. Kaner, Science, 2012, 335, 1326.

7 C. Chiang, C. Fincher Jr, Y. Park, A. Heeger, H. Shirakawa, E. Louis, S. Gau and A. G. MacDiarmid, Phys. Rev. Lett., 1977, 39, 1098.

8 S. Gabriel and J. Weiner, Ber. Dtsch. Chem. Ges., 1888, 21, 2669.

9 P. Walden, Bull. Acad. Imp. Sci. St.-Petersbourg, 1914, 8, 405.

10 W. Lu, A. G. Fadeev, B. Qi, E. Smela, B. R. Mattes, J. Ding, G. M. Spinks, J. Mazurkiewicz, D. Zhou and G. G. Wallace, Science, 2002, 297, 983.

11 V. Armel, J. Rivnay, G. Malliaras and B. Winther-Jensen, J. Am. Chem. Soc., 2013, 135, 11309.

12 M. J. Earle, J. M. S. S. Esperanca, M. A. Gilea, J. N. Canongia Lopes, L. P. N. Rebelo, J. W. Magee, K. R. Seddon and J. A. Widegren, Nature, 2006, 439, 831.

13 M. V. Fabretto, D. R. Evans, M. Mueller, K. Zuber, P. HojatiTalemi, R. D. Short, G. G. Wallace and P. J. Murphy, Chem. Mater., 2012, 24, 3998.

14 Z.-T. Zhu, J. T. Mabeck, C. Zhu, N. C. Cady, C. A. Batt and G. G. Malliaras, Chem. Commun., 2004, 1556.

15 C. Weidlich, K.-M. Mangold and K. Jüttner, Electrochim. Acta, 2005, 50, 1547.

16 W. Plieth, A. Bund, U. Rammelt, S. Neudeck and L. Duc, Electrochim. Acta, 2006, 51, 2366.

17 R. G. Pearson, J. Chem. Educ., 1968, 45, 581.

18 R. A. Marcus, Rev. Mod. Phys., 1993, 65, 599.

19 V. Gutmann, Electrochim. Acta, 1976, 21, 661.

20 F. Garnier, G. Tourillon, J. Barraud and H. Dexpert, J. Mater. Sci., 1985, 20, 2687.

21 X. Crispin, V. Geskin, A. Crispin, J. Cornil, R. Lazzaroni, W. R. Salaneck and J.-L. Brédas, J. Am. Chem. Soc., 2002, 124, 8131.

22 J. T. Mabeck and G. G. Malliaras, Anal. Bioanal. Chem., 2006, 384, 343.

23 M. Nikolou and G. G. Malliaras, Chem. Rec., 2008, 8, 13.

24 D. R. MacFarlane, J. M. Pringle, P. C. Howlett and M. Forsyth, Phys. Chem. Chem. Phys., 2010, 12, 1659.

25 O. Bubnova, Z. U. Khan, H. Wang, S. Braun, D. Evans, M. Fabretto, P. Hojati-Talemi, D. Dagnelund, J.-B. Arlin, Y. Geerts, S. Desbief, D. Breiby, J. W. Andreasen, R. Lazzaroni, W. Chen, I. Zozoulenko, M. Fahlman, P. Murphy, M. Berggren and X. Crispin, Nat. Mater., 2014, 13, 190.

26 B. Winther-Jensen, O. Winther-Jensen, M. Forsyth and D. R. MacFarlane, Science, 2008, 321, 671.

27 P. Cottis, D. Evans, M. Fabretto, S. Pering, P. J. Murphy and P. Hojati-Talemi, RSC Adv., 2014, 4, 9819.

28 E. M. Stewart, M. Fabretto, M. Mueller, P. J. Molino, H. J. Griesser, R. D. Short and G. G. Wallace, Biomater. Sci., 2013, $1,368$.

29 O. Bubnova, Z. U. Khan, A. Malti, S. Braun, M. Fahlman, M. Berggren and X. Crispin, Nat. Mater., 2011, 10, 429.

30 P. Hojati-Talemi, D. R. Evans and M. V. Fabretto, Chem. Mater., 2013, 25, 1837. 
31 L. Lindell, A. Burquel, F. L. E. Jakobsson, V. Lemaur, M. Berggren, R. Lazzaroni, J. Cornil, W. R. Salaneck and X. Crispin, Chem. Mater., 2006, 18, 4246.

32 E. Smela and N. Gadegaard, J. Phys. Chem. B, 2001, 105, 9395.

33 J. Ding, D. Zhou, G. Spinks, G. Wallace, S. Forsyth, M. Forsyth and D. MacFarlane, Chem. Mater., 2003, 15, 2392.

34 H. Tokuda, S. Tsuzuki, M. A. B. H. Susan, K. Hayamizu and M. Watanabe, J. Phys. Chem. B, 2006, 110, 19593.

35 M. N. Kobrak and H. Li, Phys. Chem. Chem. Phys., 2010, 12, 1922.

36 Y. Wang and G. A. Voth, J. Am. Chem. Soc., 2005, 127, 12192.

37 U. H. Choi, A. Mittal, T. L. Price Jr, H. W. Gibson, J. Runt and R. H. Colby, Macromolecules, 2013, 46, 1175.

38 R. Brooke, D. Evans, P. Hojati-Talemi, P. Murphy and M. Fabretto, Eur. Polym. J., 2014, 51, 28.

39 M. Fabretto, T. Vaithianathan, C. Hall, J. Mazurkiewicz, P. C. Innis, G. G. Wallace and P. Murphy, Electrochim. Acta, 2008, 53, 2250.

40 D. Mecerreyes, R. Marcilla, E. Ochoteco, H. Grande, J. A. Pomposo, R. Vergaz and J. M. Sánchez Pena, Electrochim. Acta, 2004, 49, 3555.
41 P. M. Beaujuge and J. R. Reynolds, Chem. Rev., 2010, 110, 268.

42 A. A. Argun, P. H. Aubert, B. C. Thompson, I. Schwendeman, C. L. Gaupp, J. Hwang, N. J. Pinto, D. B. Tanner, A. G. MacDiarmid and J. R. Reynolds, Chem. Mater., 2004, 16, 4401.

43 S. Liu, D. G. Kurth, H. Möhwald and D. Volkmer, Adv. Mater., 2002, 14, 225.

44 G. A. Snook, P. Kao and A. S. Best, J. Power Sources, 2011, 196, 1.

45 J. M. D’Arcy, M. F. El-Kady, P. P. Khine, L. Zhang, S. H. Lee, N. R. Davis, D. S. Liu, M. T. Yeung, S. Y. Kim, C. L. Turner, A. T. Lech, P. T. Hammond and R. B. Kaner, ACS Nano, 2014, 8, 1500.

46 Y. Yang, L. Zhang, S. Li, W. Yang, J. Xu, Y. Jiang and J. Wen, J. Mater. Sci.: Mater. Electron., 2013, 24, 2245.

47 D. R. MacFarlane, N. Tachikawa, M. Forsyth, J. M. Pringle, P. C. Howlett, G. D. Elliott, J. H. Davis, M. Watanabe, P. Simon and C. A. Angell, Energy Environ. Sci., 2014, 7, 232.

48 W. Wang, Q. Luo, B. Li, X. Wei, L. Li and Z. Yang, Adv. Funct. Mater., 2013, 23, 970. 